\title{
A Novel Three-loop Parallel Robot with Full Mobility: Kinematics, Singularity, Workspace and Dexterity Analysis
}

\author{
Wei Li* \\ PhD candidate \\ Department of Mechanical Engineering \\ and Centre for Intelligent Machines \\ McGill University \\ Montreal, QC, H3A 0C3 Canada \\ Email: livey@cim.mcgill.ca \\ Jorge Angeles \\ Professor, Fellow of ASME \\ Department of Mechanical Engineering \\ and Centre for Intelligent Machines \\ McGill University \\ Montreal, QC, H3A 0C3 Canada \\ Email: angeles@cim.mcgill.ca
}

\begin{abstract}
A novel parallel robot, dubbed the SDelta, is the subject of this paper. SDelta is a simpler alternative to both the well-known Stewart-Gough platform (SGP) and current three-limb, full-mobility parallel robots, as it contains fewer components and all its motors are located on the base. This reduces the inertial load on the system, making it a good candidate for high-speed operations. SDelta features a symmetric structure; its forward-displacement analysis leads to a system of three quadratic equations in three unknowns, which admits up to eight solutions, or half the number of those admitted by the SGP. The kinematic analysis, undertaken with a geometrical method based on screw theory, leads to two Jacobian matrices, whose singularity conditions are investigated. Instead of using the determinant of a $6 \times 6$ matrix, we derive one simple expression that characterizes the singularity condition. This approach is also applicable to a large number of parallel robots whose six actuation wrench axes intersect pairwise, such as the SGP and three-limb parallel robots whose limbs include, each, a passive spherical joint. The workspace is analyzed via a geometric method, while the dexterity analysis is conducted via discretization. Both show that the given robot has the potential to offer both large workspace and good dexterity with a proper choice of design variables.
\end{abstract}

Keywords: three-limb parallel robots, hybrid robots, full mobility, cylindrical actuators, singularity, workspace, dexterity, parallel-kinematics machine (PKM)

\section{Introduction}

A parallel robot, a.k.a. a parallel-kinematics machine (PKM), is defined as a multi-degree-of-freedom (multi-dof) articulated mechanical system composed of one moving platform (MP) and one base platform (BP), connected by at least two serial limbs [1]. Compared with their serial counterparts, PKMs offer many attractive features, as per the pertinent literature [1-3]. On the other hand, PKMs suffer of limited workspace, multiple singularities and coupled motion ${ }^{1}$, which makes their control especially challenging. These issues have motivated extensive research on PKMs.

${ }^{*}$ Corresponding author.

${ }^{1}$ This means that pure rotation or pure translation cannot be produced by a subset of the actuators. 
Early works on six-dof PKMs are found mostly around the Stewart-Gough platform (SGP) [4, 5], whose MP and BP are connected via six limbs. This number, however, results in risk of interference, complex singularity loci and limited workspace. Despite these shortcomings, most six-dof PKMs in industry still utilize this structure nowadays. Various researchers have proposed different designs with a lower number of limbs and different actuation schemes as alternative structures. Among these, three-limb, six-dof symmetric PKMs are probably the most promising candidates in applications where six-dof mobility, homogeneous performance and relatively large workspaces are required [6,7]. Numerous three-limb robots of this kind can be found in the literature [7-17]; however, when the number of limbs is smaller than six, each limb has to be actuated by more than one motor, and hence, most of these designs have some motors mounted on moving links, which increases the inertia of the system, thereby affecting its dynamic performance. In this paper we propose a novel, simple three-limb symmetric parallel robot with a special 3-CPS topology ${ }^{2}$, dubbed the SDelta Robot $^{3}$; more precisely, the robot bears a hybrid topology. The main structure is a 3-CPS PKM, each limb containing a parallel sub-structure as well, thereby making all six motors base-mounted, thus realizing a simple structure. This leads in turn to simple analysis and control, a large workspace, and low inertia, when compared with known three-limb PKMs.

Forward displacement analysis is needed in design and path-planning. According to Merlet [2], methods of analysis mainly resort to polynomial continuation [18], Gröbner bases [19] and dialytic elimination [20,21]. Polynomial continuation is capable of providing, numerically, all the solutions of the given problem, which can be used to solve very large systems, its main disadvantage being its high computational complexity. Gröbner bases offer currently the fastest method to solve the forward displacement problem in a guaranteed manner [22], but this approach can be applied only when the coefficients of the equations are rational. Regarding dialytic elimination, the system of algebraic ${ }^{4}$ equations is reduced to one univariate polynomial equation upon eliminating one unknown at a time. Elimination methods allow for the study of the properties of the solutions, even though they generally require ad hoc algebraic manipulations. We adopt an elimination method for the analysis of the SDelta Robot.

Singularity is one of the major issues arising in PKM analysis and design. Unlike serial robots, PKMs usually possess extremely complex singularity loci ${ }^{5}$ within their workspace. At singular configurations, a PKM loses or gains degrees of freedom locally, while losing either isostaticity or mobility, or encountering extreme internal forces, thereby leading to uncontrollable motion, poor performance and even damage of the mechanism. There exist four general approaches for singularity analysis, based on: screw theory [23]; Grassmann geometry [24]; differential geometry [25]; and the singularity condition of the Jacobian matrices [26,27]. Furthermore, when a six-dof parallel robot produces six actuation wrenches with axes intersecting pairwise, the singularity condition leads to a straightforward geometrical interpretation, besides a simpler formulation [28-30]. Many six-dof parallel robots, including most of the three-limb PKMs with limbs including, each, a spherical joint (see, for example, [29,31]), are designed with the foregoing feature. Based on this interpretation, we use a simpler formulation to derive one single, simple expression for the singularity condition. This condition is applicable to a large class of parallel robots whose six wrench axes intersect pairwise, i.e., most three-limb six-dof PKMs-more specifically, those with one passive spherical joint somewhere in each limb-and the SGPs whose six limbs have three attachment points on the MP.

Workspace is one of the most important attributes of PKMs. Since the workspace for a six-dof PKM is embedded in a six-dimensional space that cannot be represented graphically, one usually investigates its 3D subsets by fixing three of the six Cartesian coordinates of the MP, such as the constant-orientation workspace, reachable workspace, dexterous workspace, orientation workspace, etc. [2]. Merlet [2] classified the pertinent methods into geometrical [32,33], discretization [13,34-36] and numerical [37-39]. Geometrical methods are usually much faster, more accurate and simpler to implement for some applications, such as calculating the workspace volume [2], although they may be difficult to apply when the architecture of the PKM is complex; discretization methods have been favored for their simplicity and capacity of handling all the constraints [13,34-36]. These methods, however, are quite intensive computationally, not applicable to cases involving voids inside the workspace; numerical methods also suffer of extreme complexity when the number of constraints is large. A class of such methods, based on either interval analysis or the branch-and-prune technique have been proposed [27,40], which are capable of producing the workspace for general architectures, although they are also, in general, computationally expensive.

Different applications may impose requirements on different aspects, such as workspace volume, dexterity, stiffness and accuracy; in order to evaluate the performance in these aspects, various indices have been proposed. A comprehensive review of performance indices appeared recently [41], where the authors reviewed the existing performance indices, discussed their classification, scope and inherent limitations. The dexterity characterizes the accuracy of the robot and its homogeneity along different directions of the six-dimensional Cartesian (or joint) space [41,42]; as a result, dexterity analysis is crucial in terms of robot design and analysis; this analysis is given due attention in this paper.

\footnotetext{
${ }^{2} \mathrm{R}, \mathrm{P}, \mathrm{H}, \mathrm{C}$ and $\mathrm{S}$ denote revolute, prismatic, screw, cylindrical and spherical joints, respectively, underlined symbols denoting an actuated joint.

${ }^{3} \mathrm{~A}$ preliminary version of the SDelta robot was disclosed in an oral presentation at the Third International Workshop on Fundamental Issues and Future Research Directions for Parallel Mechanisms and Manipulators (Parallel 2014), in Tianjin, China.

${ }^{4}$ i.e., mutivariate polynomials.

${ }^{5}$ Also referred to as singularity surfaces in the literature.
} 


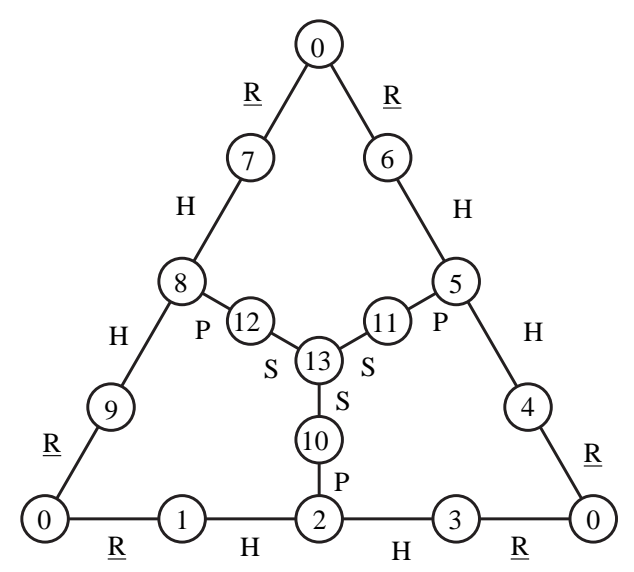

Fig. 1: Topology of the SDelta robot

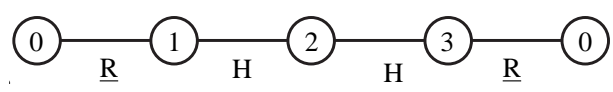

Fig. 2: Topology of the C-drive

A special 3-CPS robot, dubbed SDelta ${ }^{6}$, together with its kinematic and singularity analyses, is discussed. The novel architecture is described in Section 2. Sections 3 and 4 pertain to the forward-displacement and kinematic analyses, respectively. In Section 5, the singularity analysis is provided, which leads to a compact formulation. Sections 6 and 7 pertain to the workspace and dexterity analyses; Section 8 includes a discussion on the performance, and Section 9 summarizes the paper and provides concluding remarks.

\section{The SDelta Architecture}

First and foremost, what distinguishes the SDelta from other three-limb (or three-loop) parallel robots is its topology, as it is based on three parallel actuation mechanisms, each with a RHHR topology [43], as displayed in Figs. 1 and 2. In these figures, 0 denotes the BP, while the closed subchain 0-1-2-3-0 denotes one of the three actuation mechanisms, one on each side of the equilateral triangle of Fig. 1. In these subchains, 2 is the driving link of one limb, 5 and 8 those of the two other limbs. Moreover, 1 and 3 denote the "left" and the "right" screws driven by corresponding rotary motors. Moreover, 10, 11 and 12 denote the links of the passive C-joints making up each limb, which are coupled to the MP (13) by means of spherical joints.

PKMs with all actuators located on the base are preferred in some applications, such as those calling for high-speed operations, and in haptic devices. For this type of PKMs, motor weight will not add to the load on the actuation system, thus resulting in lower inertial loads, higher load-carrying capacity and better dynamic characteristics. Designers have come up with different solutions: Sorli et al. [10] used three double-parallelogram mechanisms to realize this goal, but this approach resorted to many extra components, which led to extra interference. Chen et al. [11] proposed a six-dof haptic device using two-dof planetary-belt systems, but these systems unavoidably introduce belt slip and flexibility, thus making it unsuitable for high-speed or high-torque applications. One major problem with these designs is that most of the actuation systems either are extremely complex or introduce many extra moving parts, thereby exacerbating the link interference, while limiting the workspace. Many other parallel robots carrying three limbs have been proposed [7,12-16], either failing to have all actuators mounted on the base or achieving this at the expense of a complex actuation system.

Despite the vast number of designs, several common issues persist: many of the existing designs involve either too many components or complex actuation systems, which affects their performance in terms of workspace, accuracy, singularity and stability. Moreover, most of the designs have their motors mounted on moving links, which leads to a waste of installed power; higher inertia also influences robot performance in high-speed applications and the quality of the system highfrequency response. Our design, as shown in the graphs of Figs. 1 and 2, departs from current practice.

The SDelta Robot bears a special 3-ㅍS topology, each $\underline{\mathrm{C}}$ joint being realized with a $\underline{\mathrm{R} H H} \underline{\mathrm{R}}$ mechanism [43], as shown in Figs. 3 and 4. Most existing three-limb parallel robots employ three serial limbs; moreover, since each limb has to carry two motors, it is not possible to use a simple limb serial chain to locate all the motors on the base. Hence, we introduce a parallel substructure in each serial limb, i.e., the two-dof cylindrical actuator. More specifically, we use a two-dof actuator, dubbed the $C$-drive [43], as shown in Fig. 4. The C-drive carries a single-loop closed kinematic chain of the RHHR type;

${ }^{6}$ Abbreviation of $\underline{\text { Six }}$-dof Delta. 


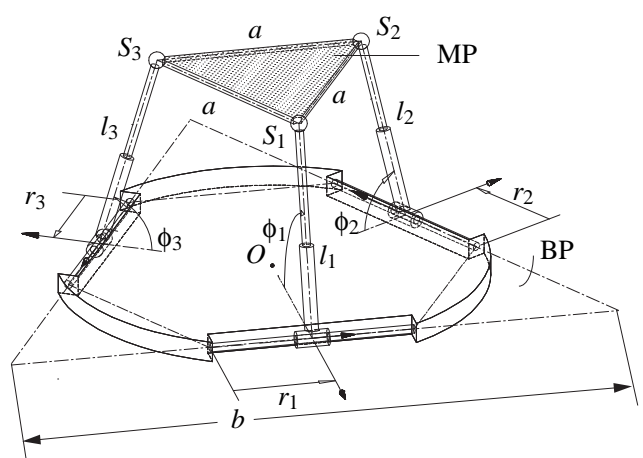

Fig. 3: Architecture of the SDelta Robot

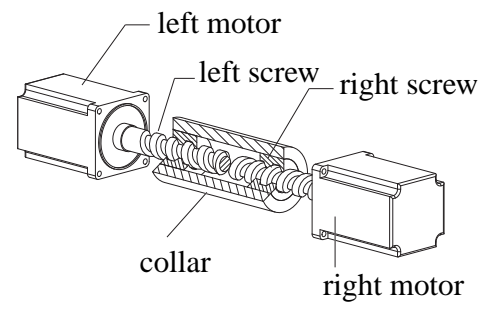

Fig. 4: An embodiment of the C-drive

the two $\mathrm{H}$ joints, of identical pitches but of opposite hands, lead to a $2 \times 2$ isotropic, constant Jacobian matrix of the drive mechanism, which is the simplest possible. The C-drive operates as a differential: when the two motors turn in the same sense $^{7}$ at the same rate, the collar undergoes a pure rotation; when the two motors turn in opposite senses at the same rate, the collar undergoes a pure translation. Each limb is thus driven in both translation along a given direction and rotation about an axis parallel to the same direction.

The SDelta Robot has the advantage that its architecture is simple, with fewer components than other three-limb designs, which reduces the complexity of its architecture and hence, simplifies its dynamics model and its control. This simplicity also reduces the potential interference among the limbs, thus resulting in a larger workspace. Moreover, mounting all motors on the base reduces the inertial load on the system, thereby making it suitable for high-speed operations. A similar topology (a 3-PRPS structure) was proposed by Behi [8], whose first two joints can be regarded as a $\mathrm{C}$ joint, but in this design, the two prismatic joints in each limb are actuated, and hence, each limb has one floating motor, significantly increasing the inertia of the system.

\section{Geometry: the Forward-displacement Analysis}

The architecture of the SDelta Robot is shown in Figs. 3 and 5, where the MP and the BP are represented by equilateral triangles, of sides $a$ and $b$, respectively. The elimination method suggested by Nanua et al. [44] is adopted for its forwarddisplacement analysis. In the foregoing paper, the authors analyzed a class of Stewart-Gough platform with three pairs of concurrent limbs, ending up with a 16th-degree resolvent polynomial; in our case, the forward-displacement problem leads to a simpler model, namely, an octic polynomial. In this vein, the actuators, i.e., the cylindrical joints, are locked, while the lengths of the limbs, $l_{j}$ for $j=1,2,3$, are unknown. Let $\mathbf{s}_{j}$ denote the position vector of point $S_{j}$, the center of the spherical joint of the $j$ th limb, for $j=1,2,3$, the relations below readily following:

$$
\left\|\mathbf{s}_{2}-\mathbf{s}_{1}\right\|^{2}=\left\|\mathbf{s}_{3}-\mathbf{s}_{2}\right\|^{2}=\left\|\mathbf{s}_{1}-\mathbf{s}_{3}\right\|^{2}=a^{2}
$$

In the forward-displacement analysis, the sliding $r_{j}$ of the $j$ th $\mathrm{C}$ joint and its rotation $\phi_{j}$ are prescribed, the pose of the MP being unknown. The MP pose is found upon locating points $S_{j}$, which is possible when the limb lengths $l_{j}$ are known, their computation being outlined below. Proceeding exactly as Nanua et al. did [44], three quadratic equations in the three limb lengths $l_{j}$ are derived:

\footnotetext{
${ }^{7}$ As viewed from the same side of the drive layout.
} 


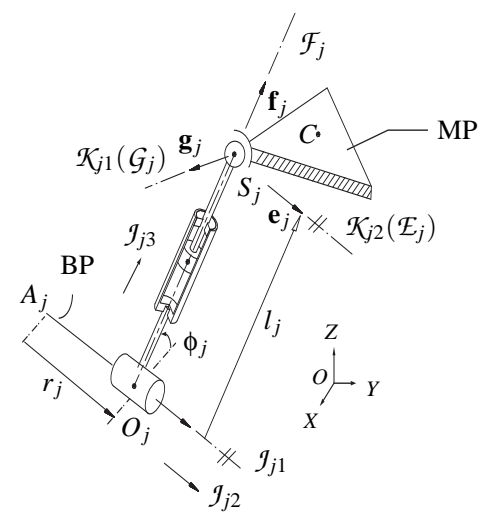

Fig. 5: Notation for the kinematic chain of the $j$ th limb of the SDelta Robot

$$
\begin{aligned}
& l_{1}^{2}+l_{2}^{2}-\sqrt{3}\left(b-r_{1}\right) \cos \phi_{2} l_{2}+b^{2}-2 b r_{1}+r_{1}^{2}+r_{2}^{2} \\
&+\left[\left(\cos \phi_{1} \cos \phi_{2}-2 \sin \phi_{1} \sin \phi_{2}\right) l_{2}-\sqrt{3} r_{2} \cos \phi_{1}\right] l_{1} \\
&+r_{1} r_{2}-b r_{2}-a^{2}=0 \\
& l_{2}^{2}+l_{3}^{2}-\sqrt{3}\left(b-r_{2}\right) \cos \phi_{3} l_{3}+b^{2}-2 b r_{2}+r_{2}^{2}+r_{3}^{2} \\
&+\left[\left(\cos \phi_{2} \cos \phi_{3}-2 \sin \phi_{2} \sin \phi_{3}\right) l_{3}-\sqrt{3} r_{3} \cos \phi_{2}\right] l_{2} \\
&+r_{2} r_{3}-b r_{3}-a^{2}=0 \\
& l_{3}^{2}+l_{1}^{2}-\sqrt{3}\left(b-r_{3}\right) \cos \phi_{1} l_{1}+b^{2}-2 b r_{3}+r_{3}^{2}+r_{1}^{2} \\
&+\left[\left(\cos \phi_{1} \cos \phi_{3}-2 \sin \phi_{1} \sin \phi_{3}\right) l_{1}-\sqrt{3} r_{1} \cos \phi_{3}\right] l_{3} \\
&+r_{3} r_{1}-b r_{1}-a^{2}=0
\end{aligned}
$$

The three foregoing equations thus admit up to $2^{3}=8$ roots. This is half the number of the solutions of the forwarddisplacement problem admitted by the SGP with triangular BP and MP.

\section{Kinematics}

\subsection{The Jacobian Matrix of the SDelta Robot}

The architecture of the SDelta Robot, whose three cylindrical drives are actuated, is shown in Figs. 3 and 5. Let $\mathbf{c}$ be the position vector of the operation point $C$ on the moving platform ${ }^{8}, \mathbf{t}=\left[\boldsymbol{\omega}^{T} \dot{\mathbf{c}}^{T}\right]^{T}$ the twist of the MP, with $\boldsymbol{\omega}$ denoting the

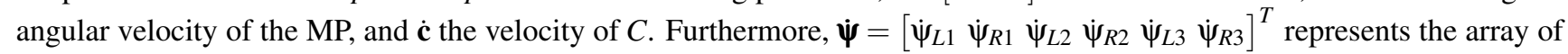
six motor rates, three to the right $(R)$, three to the left $(L)$ of point $O_{j}$ of the C-Drive collar in Fig. 5, for $j=1,2,3$. Then we need to find the mapping between $\mathbf{t}$ and $\boldsymbol{\psi}$. It is known [32] that the array of actuated-joint rates $\boldsymbol{\psi}$ and the MP twist are related by two Jacobian matrices, $\mathbf{J}$ and $\mathbf{K}$, namely,

$$
\mathbf{K t}=\mathbf{J} \dot{\psi}
$$

However, since the C-drive of the SDelta Robot carries, each, two screw pairs, the derivation of its Jacobian matrices is not as straightforward. We thus introduce a new array:

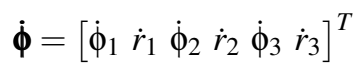

where $\dot{\phi}_{j}, \dot{r}_{j}$ represent the turning and the sliding rates of the collar of the $j$ th C-drive, for $j=1,2,3$. Now we can express Eqn. (3) in the form

$$
\mathbf{K} \mathbf{t}=\mathbf{D} \dot{\boldsymbol{\phi}}, \quad \dot{\boldsymbol{\phi}}=\mathbf{J}_{m} \dot{\boldsymbol{\psi}}
$$




$$
\mathbf{J}=\mathbf{D} \mathbf{J}_{m}
$$

\subsubsection{The $K$ and $D$ Matrices}

Firstly we introduce our notation: Vector $\mathbf{p}_{j}$ is defined as that joining the $j$ th spherical joint center $S_{j}$ with the operation point $C$, while $\mathbf{e}_{j}$ and $\mathbf{f}_{j}$, for $j=1,2,3$, all shown in Fig. 5, represent the unit vectors associated with the $j$ th C-drive axis and the $j$ th-limb axis; $\mathbf{g}_{j}$ is defined as the direction vector along $\mathbf{e}_{j} \times \mathbf{f}_{j}$. It is noteworthy that $\left\{\mathbf{e}_{j}, \mathbf{f}_{j}, \mathbf{g}_{j}\right\}$ is an orthonormal, right-handed triad, as illustrated in Fig. 5.

Matrices $\mathbf{K}$ and $\mathbf{D}$ relate the end-effector twist with the array of the turning and sliding rates of the collars of the three C-drives. These Jacobian matrices can be conveniently derived based on screw theory [23,45]. It is known that every joint in the robot bears an axis (i.e., a finite line or a line at infinity) associated with a corresponding array of Plücker coordinates; in this vein, let us assume that the $i$ th joint variable of $\boldsymbol{\phi}$ is associated with the $j$ th joint of the $k$ th limb; then, the $i$ th row of $\mathbf{K}$ must be a screw ${ }^{9}$ that is reciprocal [46] to the Plücker coordinates of all the axes in the $k$ th limb, but the one associated with the $i$ th component of $\phi$. Moreover, it is recalled that a finite line can be regarded as a zero-pitch screw, while a line at infinity as an infinite-pitch screw. Furthermore,

(a) Two zero-pitch screws are reciprocal when they are coplanar.

(b) Two infinite-pitch screws are always reciprocal.

(c) A zero-pitch screw is reciprocal to an infinite-pitch screw when their directions are orthogonal.

Now consider line $\mathcal{K}_{j 1}$, associated with the wrench corresponding to the rotational degree of freedom of the $j$ th C-drive. Since there exists a spherical joint at $S_{j}, \mathcal{K}_{j 1}$ must pass through $S_{j}$; moreover, its direction vector must be normal to the direction vector of $\mathcal{g}_{j 2}$ and $\mathcal{I}_{j 3}$. Thus, $\mathcal{K}_{j 1}$ passes through $S_{j}$, with its direction vector normal to $\mathbf{e}_{j}$ and $\mathbf{f}_{j}$, i.e., along $\mathbf{g}_{j}$, as shown in Fig. 5.

Next, consider line $\mathcal{K}_{j 2}$, associated with the wrench corresponding to the translational degree of freedom of the $j$ th Cdrive. Since $\mathcal{K}_{j 2}$ passes through $S_{j}$, it is coplanar with $\mathcal{I}_{j 1}$, and normal to $\mathfrak{I}_{j 3}$; we can then conclude that $\mathcal{K}_{j 2}$ passes through $S_{j}$ and is parallel to the axis of the $j$ th C-drive, as shown, again, in Fig. 5. Based on the above analysis, we can obtain the $\mathbf{K}$ matrix as

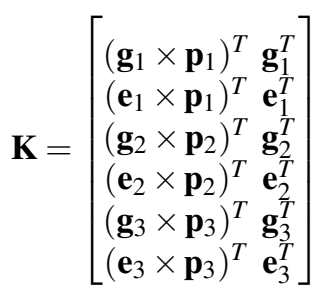

Under this notation, $\mathbf{g}_{j}$ and $\mathbf{e}_{j}$ represent the unit vectors parallel to lines $\mathcal{K}_{j 1}$ and $\mathcal{K}_{j 2}$, respectively, for $j=1,2,3$. We will henceforth denote $\mathcal{K}_{j 1}$ and $\mathcal{K}_{j 2}$ as $\mathcal{G}_{j}$ and $\mathcal{E}_{j}$, respectively, for simplicity, as shown in Fig. 5, their Plücker arrays being $\mathbf{k}_{j 1}=\left[\mathbf{g}_{j}^{T},\left(\mathbf{g}_{j} \times \mathbf{p}_{j}\right)^{T}\right]^{T}$ and $\mathbf{k}_{j 2}=\left[\mathbf{e}_{j}^{T},\left(\mathbf{e}_{j} \times \mathbf{p}_{j}\right)^{T}\right]^{T}$.

Once $\mathbf{K}$ is available, $\mathbf{D}$ is straightforward to derive as a diagonal matrix, whose $i$ th component is the reciprocal product of the line corresponding to the $i$ th row of $\mathbf{K}$ and that associated with the joint of the $i$ th component of $\boldsymbol{\phi}$. $\mathbf{D}$ is found to bear the form

$$
\mathbf{D}=\operatorname{diag}\left(l_{1}, 1, l_{2}, 1, l_{3}, 1\right)
$$

with $l_{i}$ representing the length of the $i$ th limb, thereby deriving the two Jacobian matrices, $\mathbf{K}$ in Eqn. (7) and $\mathbf{D}$ in Eqn. (8).

\subsubsection{The $\mathbf{J}_{m}$ Matrix}

Matrix $\mathbf{J}_{m}$ relates the array of six motor rates with the array of the turning and sliding rates of the collars of the three C-drives. The Jacobian matrix $\mathbf{J}_{C}$ of the C-drive [43] relates the speeds of the two screws with the turning and sliding rates

\footnotetext{
${ }^{9}$ In its ray coordinates
} 
of the collar, namely:

$$
\dot{\boldsymbol{\gamma}}_{C}=\mathbf{J}_{C} \dot{\boldsymbol{\Psi}}_{C}
$$

with

$$
\dot{\boldsymbol{\gamma}}_{C}=\left[\begin{array}{l}
\dot{\phi} \\
\dot{r}
\end{array}\right], \mathbf{J}_{C}=\left[\begin{array}{cc}
1 / 2 & 1 / 2 \\
p / 4 \pi & -p / 4 \pi
\end{array}\right], \dot{\boldsymbol{\Psi}}_{C}=\left[\begin{array}{c}
\dot{\psi}_{L} \\
\dot{\psi}_{R}
\end{array}\right]
$$

where $\dot{\gamma}_{C}$ and $\dot{\psi}_{C}$ represent the speeds of the collar and of the screws, respectively, $\dot{\psi}_{L}, \dot{\psi}_{R}$ the angular speeds of the left and right screws of the C-drive, respectively, and hence, of the C-drive motors.

It is noteworthy that the three C-drives are identical, the above relation thus applying to all of them. Apparently, then $\mathbf{J}_{m}$ is a block-diagonal matrix for the three $\mathrm{C}$-drives:

$$
\mathbf{J}_{m}=\operatorname{diag}\left(\mathbf{J}_{C}, \mathbf{J}_{C}, \mathbf{J}_{C}\right)
$$

thereby obtaining relation (5).

\subsubsection{Redefinition of $\mathbf{J}_{m}$}

If the rates of the C-drive are redefined as $\dot{\gamma}_{C}=\left[\dot{\phi}^{T}, 2 \pi \dot{r} / p^{T}\right]^{T}$, then, we can rewrite Eqn. (9) as

$$
\dot{\boldsymbol{\gamma}}_{C}=\mathbf{J}_{C} \dot{\boldsymbol{\Psi}}_{C} \text {, with } \mathbf{J}_{C}=\left[\begin{array}{cc}
1 / 2 & 1 / 2 \\
1 / 2 & -1 / 2
\end{array}\right], \dot{\boldsymbol{\Psi}}_{C}=\left[\begin{array}{l}
\dot{\psi}_{L} \\
\dot{\psi}_{R}
\end{array}\right]
$$

In this vein, we redefine $\mathbf{J}_{m}$ as

$$
\mathbf{J}_{m}=\operatorname{diag}\left(\mathbf{J}_{C}, \mathbf{J}_{C}, \mathbf{J}_{C}\right)
$$

which is a constant isotropic matrix because $\mathbf{J}_{C}$ in Eqn. (12) is isotropic. Correspondingly, $\mathbf{D}$ is redefined as

$$
\mathbf{D}=\operatorname{diag}\left(l_{1}, \frac{p}{2 \pi}, l_{2}, \frac{p}{2 \pi}, l_{3}, \frac{p}{2 \pi}\right)
$$

in order to preserve the equality in Eqn. (6). Finally, the $\mathbf{J}$ matrix becomes

$$
\mathbf{J}=\mathbf{D} \mathbf{J}_{m}
$$

where $\mathbf{D}$, as displayed in Eqn. (14), is a diagonal matrix whose entries have all units of length, while $\mathbf{J}_{m}$ is a $6 \times 6$ dimensionless block-diaongal isotropic matrix.

\section{Singularity Analysis}

The singularities of parallel robots pertain to 1) those occurring in the serial Jacobian matrices $\mathbf{J}_{j}$ of any of the limbs and 2) those occurring in matrices $\mathbf{K}$ and $\mathbf{D}$. The latter are what are known as type-I (for $\mathbf{K}$ ) and type-II for (D) singularities, respectively [32].

\subsection{Singularities of the Serial Jacobian Matrices}

Since each limb of the SDelta Robot has a decoupled architecture-i.e., a spherical joint coupling the MP with each limb - the singularity analysis of their Jacobian matrices is straightforward. The pertinent singularities can be classified into 
wrist and shoulder singularities: Wrist singularities occur when the three wrist axes become coplanar. This means that the axes of the three concurrent revolute joints are coplanar; the shoulder singularity occurs when point $C$ lies in the hyperboloid defined by the first three limb axes [47]. This condition means that there exists one line $\mathcal{L}_{j}$ passing through $S_{j}$ that is coplanar with $\mathcal{I}_{j 1}$, normal to $\mathcal{I}_{j 2}$ and $\mathcal{I}_{j 3}$. This can only happen when the length of the $j$ th limb is zero, which is physically impossible for the SDelta architecture.

\subsection{Type-I Singularity}

Since $\mathbf{D}$ is a diagonal matrix, it becomes singular when any of its diagonal entries vanishes. From Eqn. (14) it can be readily seen that this happens when the length of one of the limbs is zero, which is, again, physically impossible in general. This condition is the same as that for the shoulder singularity of serial Jacobian matrices, and hence, excluded.

\subsection{Type-II Singularity}

This occurs when $\mathbf{K}$ becomes singular, and the robot gains extra mobility ${ }^{10}$. The singularity of three-limb PKMs with one passive $\mathrm{S}$ joint at each limb end has been investigated extensively [31,48-51], based on: instability analysis [31]; screw theory [48]; passive joint velocities [49]; the pure condition [50]; and the characteristic tetrahedron [51]. Moreover, the singularity of a more general class of PKMs, namely, three-limb PKMs whose limbs, each, includes a passive S joint somewhere, has also been investigated based on passive joint velocities [28] and Grassmann-Cayley algebra [29, 30]. It has been shown that the singularity of this class of robots yields a straightforward geometrical interpretation, namely, the four planes - three planes composed by the three pairs of intersecting wrench axes plus the plane of the MP triangle-share at least one common point [28-30]. Starting from this geometric condition, we propose a simple formulation for the singularity condition without involving any determinant calculation [28,31,49-51] or passive joint velocities [28, 49].

We have obtained $\mathbf{K}$ as shown in Eqn. (7), whose six rows can be regarded as the Plücker coordinates of six actuated wrenches, intersecting pairwise at the center of the three spherical joints. Since these six wrench lines of $\mathbf{K}$ intersect in three pairs in our case, it has been found that $\mathbf{K}$ becomes singular when the four planes-three planes composed by the three pairs of intersecting wrench lines together with the plane of the MP triangle - share at least one common point [28-31,50,51]. We denote the plane defined by the intersecting lines $\mathcal{E}_{j}, \mathcal{G}_{j}$ as $\Pi_{j}$, its normal as $\mathbf{n}_{j}$, for $j=1,2,3$. It is noteworthy that $\mathbf{n}_{j}=\mathbf{f}_{j}$ for the SDelta Robot, but we use $\mathbf{n}_{j}$ for generality. Moreover, we denote the MP plane as $\Pi_{4}$, its normal as $\mathbf{n}_{4}$. Next we conduct the singularity analysis based on this geometrical interpretation.

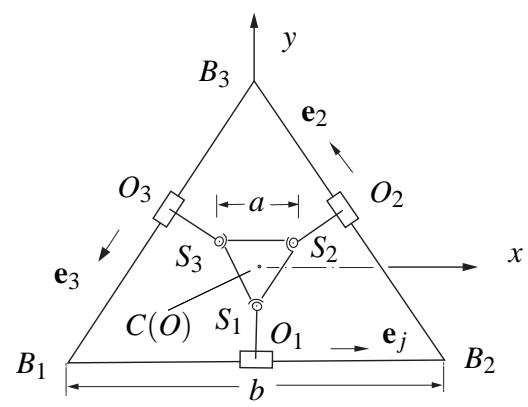

Fig. 6: The reference posture of the MP

First, denote the intersecting line between $\Pi_{j}$ and $\Pi_{4}$ as $\mathcal{L}_{j}$, for $j=1,2,3$; it is noteworthy that $\mathcal{L}_{j}$ passes through $S_{j}$, and lies in the common plane $\Pi_{4}$; the foregoing geometrical condition is then equivalent to requiring that the three lines $\mathcal{L}_{j}$, for $j=1,2,3$, share common points. In this way, we can reduce the analysis to the plane $\Pi_{4}$. Furthermore, let us denote the intersecting point of $\mathcal{L}_{1}$ with $\mathcal{L}_{2}$ and with $\mathcal{L}_{3}$ as $R_{2}$ and $R_{3}$, respectively; then, the condition leads to requiring that the position vectors of $R_{2}$ and $R_{3}$ be identical.

Let us denote the direction vector of $\mathcal{L}_{j}$ as $\mathbf{l}_{j}$; then, $\mathbf{l}_{j}$ must be normal to both $\mathbf{n}_{j}$ and $\mathbf{n}_{4}$. We do not require $\mathbf{l}_{j}$ to be of unit norm here, and hence, we can assign

$$
\mathbf{l}_{j}=\mathbf{n}_{j} \times \mathbf{n}_{4} \quad j=1,2,3
$$

\footnotetext{
${ }^{10}$ In the ensuing derivation, $\mathbf{p}_{j}$ denotes the position vector pointing from $S_{j}$, the $j$ th spherical joint center to the operation point $C$ on the MP, these centers being the vertices of the MP when the three spherical joints are connected directly to the MP, as in our case.
} 
Furthermore, upon denoting the position vector of the common point as $\mathbf{r}$, we have

$$
\mathbf{r}=k_{1} \mathbf{l}_{1}-\mathbf{p}_{1}=k_{2} \mathbf{l}_{2}-\mathbf{p}_{2}=k_{3} \mathbf{l}_{3}-\mathbf{p}_{3}
$$

where $k_{j}, j=1,2,3$, are as yet to be determined. From the above relations, we have

$$
\begin{aligned}
& k_{1} \mathbf{l}_{1}-k_{2} \mathbf{l}_{2}=\mathbf{p}_{1}-\mathbf{p}_{2} \\
& k_{1} \mathbf{l}_{1}-k_{3} \mathbf{l}_{3}=\mathbf{p}_{1}-\mathbf{p}_{3}
\end{aligned}
$$

Next we cross-multiply both sides of Eqn. (18a) with $\mathbf{l}_{2}$, of Eqn. (18b) with $\mathbf{l}_{3}$, which leads to

$$
\begin{aligned}
& k_{1} \mathbf{l}_{1} \times \mathbf{l}_{2}=\left(\mathbf{p}_{1}-\mathbf{p}_{2}\right) \times \mathbf{l}_{2} \\
& k_{1} \mathbf{l}_{1} \times \mathbf{l}_{3}=\left(\mathbf{p}_{1}-\mathbf{p}_{3}\right) \times \mathbf{l}_{3}
\end{aligned}
$$

It can be seen that both sides of Eqns. (19a) and (19b) are parallel to the $z$-axis of the MP frame. Next, upon dot-multiplying the LHS of Eqn. (19a) with the RHS of Eqn. (19b), then equating this product with that of the corresponding sides of Eqns. (19a) and (19b), we obtain

$$
\left(\mathbf{l}_{1} \times \mathbf{l}_{2}\right) \cdot\left[\left(\mathbf{p}_{1}-\mathbf{p}_{3}\right) \times \mathbf{l}_{3}\right]=\left(\mathbf{l}_{1} \times \mathbf{l}_{3}\right) \cdot\left[\left(\mathbf{p}_{1}-\mathbf{p}_{2}\right) \times \mathbf{l}_{2}\right]
$$

where the common factor $k_{1}$ has been eliminated. Moreover, plugging Eqn. (16) into Eqn. (20), after some manipulations, leads to

$$
\begin{array}{r}
{\left[\left(\mathbf{n}_{3}-\mathbf{n}_{3} \cdot \mathbf{n}_{4} \mathbf{n}_{4}\right) \cdot\left(\mathbf{p}_{1}-\mathbf{p}_{3}\right)\right]\left[\left(\mathbf{n}_{2} \times \mathbf{n}_{1}\right) \cdot \mathbf{n}_{4}\right]=} \\
\quad\left[\left(\mathbf{n}_{2}-\mathbf{n}_{2} \cdot \mathbf{n}_{4} \mathbf{n}_{4}\right) \cdot\left(\mathbf{p}_{1}-\mathbf{p}_{2}\right)\right]\left[\left(\mathbf{n}_{3} \times \mathbf{n}_{1}\right) \cdot \mathbf{n}_{4}\right]
\end{array}
$$

Furthermore, it is noted that $\mathbf{n}_{4}$ is normal to $\mathbf{p}_{i}-\mathbf{p}_{j}$, for $i, j=1,2,3, i \neq j$; we can thus simplify Eqn. (21) to

$$
\begin{array}{r}
{\left[\mathbf{n}_{3} \cdot\left(\mathbf{p}_{1}-\mathbf{p}_{3}\right)\right]\left[\left(\mathbf{n}_{2} \times \mathbf{n}_{1}\right) \cdot \mathbf{n}_{4}\right]=} \\
{\left[\mathbf{n}_{2} \cdot\left(\mathbf{p}_{1}-\mathbf{p}_{2}\right)\right]\left[\left(\mathbf{n}_{3} \times \mathbf{n}_{1}\right) \cdot \mathbf{n}_{4}\right]}
\end{array}
$$

which is the singularity condition sought. It is observed that, when represented in the MP frame, $\mathbf{p}_{j}$ and $\mathbf{n}_{4}$ in Eqn. (22) become constant, which reduces the computational cost greatly. As a result, we choose to express all the vectors in the MP frame. Then, we only need to find $\mathbf{n}_{j}$, for $j=1,2,3$, in the MP frame. It is noteworthy that, for the SDelta robot, $\mathbf{n}_{j}$ is nothing but $\mathbf{f}_{j}$, and hence, parallel to the axis of the $j$ th limb; moreover, its norm does not affect the relation in Eqn. (22). Assuming that the perpendicular foot of $S_{j}$ on the axis of the $j$ th C-drive is $O_{j}$, as shown in Figs. 5 and 6 , we can use $\overrightarrow{O_{j} S_{j}}$ to substitute $\mathbf{n}_{j}$ in Eqn. (22), where

$$
\overrightarrow{O_{j} S_{j}}=\left(\mathbf{1}-\mathbf{e}_{j} \mathbf{e}_{j}^{T}\right) \overrightarrow{B_{j} S_{j}}=\left(\mathbf{1}-\mathbf{e}_{j} \mathbf{e}_{j}^{T}\right)\left(\overrightarrow{C S_{j}}-\overrightarrow{C B_{j}}\right)
$$

which yields

$$
\left[\overrightarrow{O_{j} S_{j}}\right]_{\mathcal{M}}=\mathbf{Q}^{T}\left(\mathbf{1}-\left[\mathbf{e}_{j}\right]_{\mathcal{B}}\left[\mathbf{e}_{j}\right]_{\mathcal{B}}^{T}\right)\left\{[\mathbf{c}]_{\mathcal{B}}-\left[\overrightarrow{O B_{j}}\right]_{\mathcal{B}}-\mathbf{Q}\left[\mathbf{p}_{j}\right]_{\mathcal{M}}\right\}
$$

in which only $\mathbf{Q}$ and $\mathbf{c}$ are variable. Then we can use $\overrightarrow{O_{j} S_{j}}$ to substitute $\mathbf{n}_{j}$ in Eqn. (22), for $j=1,2,3$.

It can be readily verified that this equality also holds even if some of the four planes $\Pi_{j}$ are coincident, or when some of the three intersecting lines $\mathcal{L}_{j}$ coincide. 


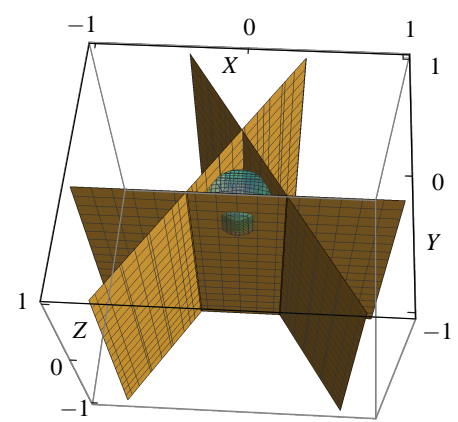

(a)

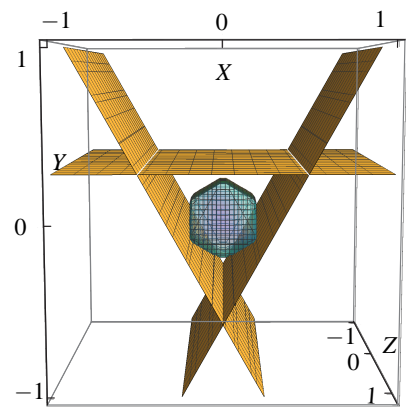

(b)

Fig. 7: The workspace and singularity of the SDelta under the reference orientation (a) Design I (b) Design II

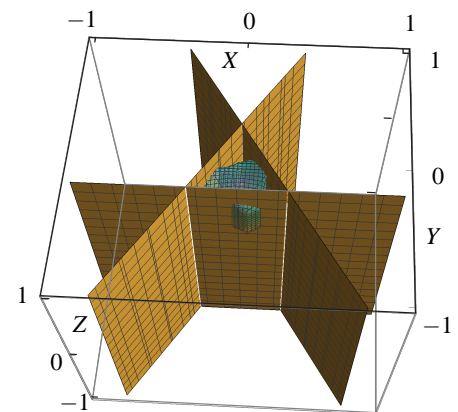

(a)

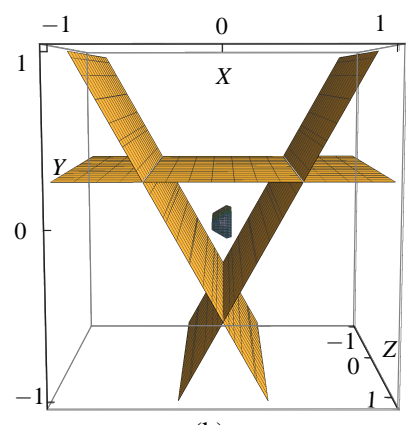

(b)

Fig. 8: The workspace and singularity of the SDelta with the orientation $\mathbf{e}=[0,0,1]^{T}$ and $\phi=15^{\circ}$ (a) Design I (b) Design II

\subsection{Case Study: the Fixed-Orientation Singularity Locus}

Since the singularity locus of a six-dof PKM is impossible to visualize, its fixed-orientation subset has been mostly investigated in the literature, which means that the orientation, i.e., the $\mathbf{Q}$ matrix, is fixed. Further, $\overrightarrow{O_{j} S_{j}}$ is linear in $\mathbf{c}$, while $\left(\mathbf{n}_{j} \times \mathbf{n}_{1}\right) \cdot \mathbf{n}_{4}$ is quadratic in $\mathbf{c}$, for $j=2,3$; it then follows that Eqn. (22) yields a cubic surface in $\mathbf{c}$. A numerical example is given below for illustration.

It is found that the design parameters impact on singularity distribution, workspace and dexterity. Hence, we plot these three items for two typical sets of design variables, namely, the sides of the MP and the BP are assumed to obey the relation $a / b=0.3$ for Design I and $a / b=1$ for Design II, respectively. Furthermore, we define the reference pose of the MP as that under which the operation point $C$ coincides with $O$ in the BP, while the MP orientation is as shown in Fig. 6, with the BP and MP planes coincident.

Several typical orientations are selected, under which the singularity loci are plotted as the surfaces shown in Figs. 7 to 9 , with the open and closed surfaces representing the singularity locus and the corresponding workspace boundary, respectively. In these figures, the orientation is given by axis $\mathbf{e}$ and angle $\phi$ of the rotation matrix that carries the BP from its reference to its current attitude. It is observed that, when the MP rotates about the $Z$-axis, the singularity is characterized by three vertical planes, which can be analyzed by means of Grassmann geometry: at the reference orientation, the three wrench axes $\mathcal{E}_{i}$ corresponding to the three C-Drive axes are coplanar, in a horizontal plane; then, when the middle link of the $i$ th limb is vertical, the wrench line corresponding to $\mathbf{g}_{i}$ lies also in the same horizontal plane, these four lines becoming a linear variety [24] of rank 3, which leads to a singular configuration. Hence, the three planes can be found when the MP translates to a configuration in which $S_{i}$ lies in the vertical plane that passes through the $i$ th C-Drive axis. Furthermore, when the MP undergoes a rotation about the $Z$-axis, the three lines $\mathcal{E}_{i}$ are still in the same horizontal plane, which again, leads to three vertical singularity planes; this is simple to characterize. However, when the MP rotates about some other axes, the singularity surface has, generally, a complex shape.

\section{Workspace Analysis}

In this section we investigate the fixed-orientation position workspace to have a general idea of the workspace volume. We consider only the limits of the active and passive joints first, then verify whether singularities exist within the workspace thus obtained.

We propose a geometrical method capable of obtaining the workspace efficiently, and thus, can be used for workspace 


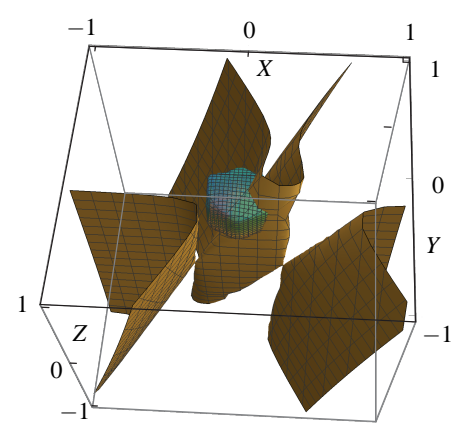

(a)

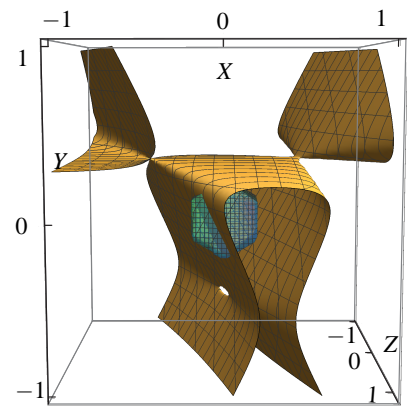

(b)

Fig. 9: The workspace and singularity of the SDelta with the orientation $\mathbf{e}=[0,1,0]^{T}$ and $\phi=15^{\circ}$ (a) Design I (b) Design II

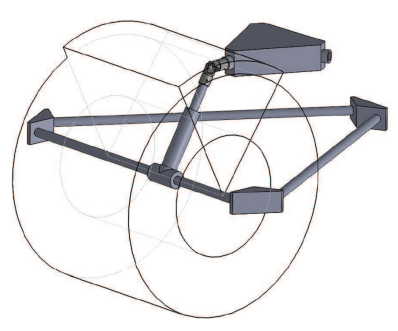

(a)

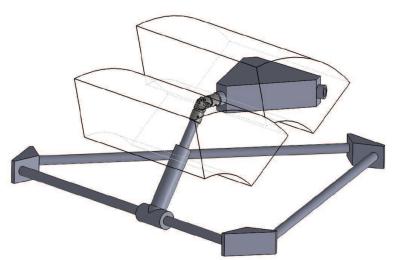

(b)

Fig. 10: Illustration of the workspace formulation based on the geometric method

optimization. The basic principle of the construction of the fixed-orientation workspace follows: We find the feasible workspace of the MP under the constraint of only the $i$ th limb (i.e., we assume that the two other limbs are disconnected and regard the robot as a serial chain), denoted as $\mathcal{W}_{i}$; then, the workspace of interest will be the intersection of $\left\{\mathcal{W}_{i}\right\}_{1}^{3}$. Next, we explain the procedure for finding $\mathcal{W}_{i}$.

Since the orientation of the MP is fixed for the fixed-orientation workspace, the center of the spherical joint $S_{i}$, undergoes the same motion as the operation point $C$. Hence, we firstly find the "position workspace" of $S_{i}$-denoted as $\mathcal{W}_{S i}$. Considering the stroke of the C-drive, denoted $r_{s}$, the upper and lower bounds of the telescopic arm, denoted $l_{\min }$ and $l_{\max }$, the position of $S_{i}$ is found to lie within the region between two co-axial cylinders of radii $l_{\min }$ and $l_{\max }$, respectively, whose height is the stroke $r_{s}$, as shown in Fig. 10(a).

Furthermore, we consider the constraint of the $\mathrm{S}$ joint, which we assume to be realized by a ball-and-socket joint, whose working angle range can be modelled as a cone [2], its maximum denoted $\delta_{\max }$. Apparently, the translation of the MP along the direction $\mathbf{e}_{i}$ of the axis of the C-drive, or along the direction $\mathbf{f}_{i}$ of the $i$ th limb, will not change the relative orientation of the two links connected by the spherical joint (i.e. the upper link of the telescopic boom and the MP); only the translation of the MP in the direction of $\mathbf{g}_{i}$ will change the foregoing relative orientation, which corresponds to the rotation of the C-drive. The motion of the $\mathrm{S}$ joint has a limit only on the feasible range of the angle of rotation of the $\mathrm{C}$-drive; this limit remains constant when $S_{i}$ translates in the direction of $\mathbf{e}_{i}$ or $\mathbf{f}_{i}$. This means that, when we further consider the limits of the $\mathrm{S}$ joint, we will no longer have a cylindrical shape, but a pie slice, as shown, again, in Fig. 10(a), whose angular range can be derived from the projection of the cone onto the plane normal to $\mathbf{e}_{i}$. Until now, we have found $\mathcal{W}_{s i}$; it is a simple matter to translate this region by $\mathbf{p}_{i}$ to find $\mathcal{W}_{i}$, the feasible region of the operation point under the constraint of the $i$ th limb, as shown in Fig. 10(b). Once all the three regions $\left\{\mathcal{W}_{i}\right\}_{1}^{3}$ are available, their intersection yields the fixed-orientation workspace sought. Researchers usually discretize one of the three coordinates, e.g., the Z-coordinate, then find the workspace shape on different layers. With the aid of computer-algebra, this kind of intersection operation of geometrical objects can be handled directly; the software in use also provides the workspace volume and the workspace boundary.

\subsection{Case Study}

The fixed-orientation worksapce is provided for a robot, again, with two sets of design parameters:

$$
\text { and } \quad \begin{aligned}
a & =0.2 b, l_{\min }=0.45 b, l_{\max }=0.85 b \\
& =b, l_{\min }=0.6 b, l_{\max }=1.13 b
\end{aligned}
$$




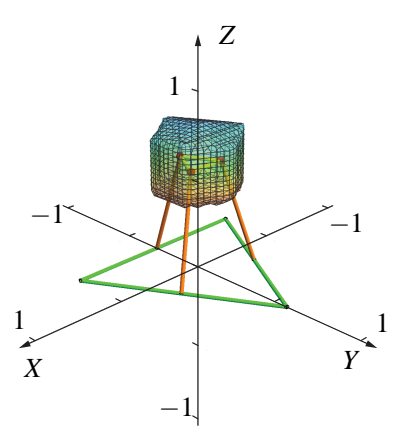

(a)

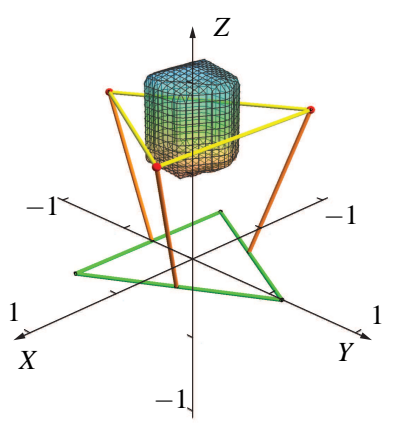

(b)

Fig. 11: The workspace of the Sdelta under the reference orientation (a) Design I (b) Design II

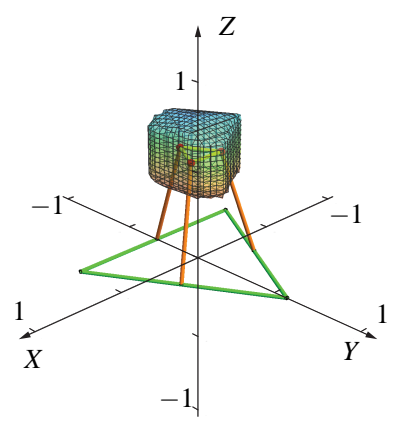

(a)

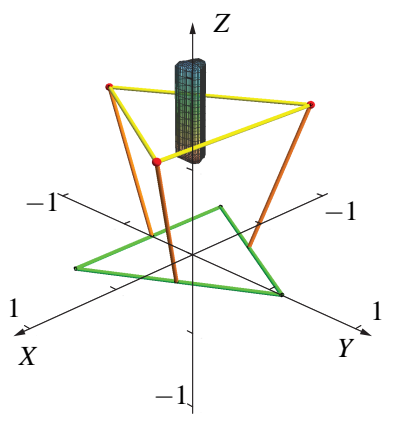

(b)

Fig. 12: The workspace of the SDelta with the orientation $\mathbf{e}=[0,0,1]^{T}$ and $\phi=15^{\circ}$ (a) Design I (b) Design II

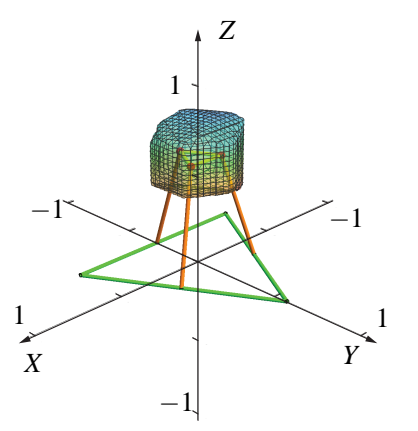

(a)

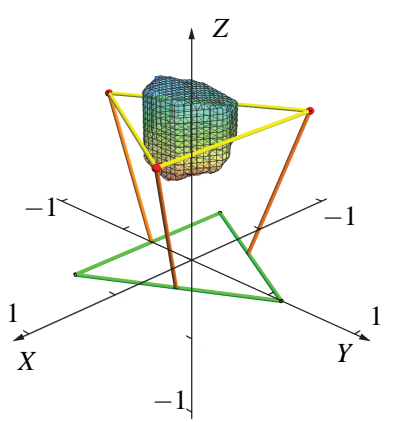

(b)

Fig. 13: The workspace of the SDelta with the orientation $\mathbf{e}=[0,1,0]^{T}$ and $\phi=15^{\circ}$ (a) Design I (b) Design II

where $l_{\min }$ and $l_{\max }$ represent the minimum and maximum lengths of each limb; It is noteworthy that we choose different $l_{\min }$ and $l_{\max }$ values in order to keep the height of the MP similar at the reference pose for the two set of different design parameters. Moreover, we assume the maximum angle attained by the $\mathrm{S}$ joint to be $\delta_{\max }=45^{\circ}$; then, the workspace under the reference orientation is plotted in Fig. 11 for each case, yielding volume values of $0.049 d^{3}$ and $0.067 b^{3}$, respectively. The workspace is also evaluated under several other orientations, as shown in Figs. 12 and 13, with the volumes $0.045 b^{3}, 0.007 b^{3}, 0.047 b^{3}, 0.042 b^{3}$, respectively. It is, however, noteworthy that the singularity surface sometimes crosses the workspace for the given set of design parameters and selected orientation, as shown in Figs. 7 to 9, similar to the case of the Stewart-Gough platform [52].

Finally, in order to reveal the effect of the ratio $a / b$ on the workspace volume $(V)$, we plot $V$ vs. $a / b$, in the range of 0.2 to 1.5 , which range we believe to be sufficient in general applications. Moreover, even though we used different $l_{\min }$ and $l_{\max }$ for Designs I and II in order to make the height of the MP similar at the reference pose, we fix them here in order to reveal the effect of solely the ratio $a / b$; more specifically, $l_{\min }=0.55 b, \quad l_{\max }=b$. Then, the workspace volumes are, again, plotted under the three different orientations, i.e., the reference orientation, the orientation $\mathbf{e}=[0,0,1]^{T}$ with $\phi=15^{\circ}$ 
and $\mathbf{e}=[0,1,0]^{T}$ with $\phi=15^{\circ}$, respectively, as shown in Fig. 14. Apparently, the workspace volume $V$ under the reference orientation remains largely unchanged for different values of $a / b$; however, $V$ decreases significantly when the MP is rotated about the Z-axis; when the MP is rotated about other directions, the workspace volume can either decrease or increase. It is noteworthy that the above workspace volume is different from those of Designs I and II, indicating that the range of the passive limb length affects the workspace as well.

The above result indicates that, when the range of the passive limb length is fixed, the workspace volume generally decreases as the ratio $a / b$ increases; however, for a larger MP, (e.g., $a=b)$, the robot allows for a larger range of the passive limb length ${ }^{11}$, which can end up with a bigger workspace volume.

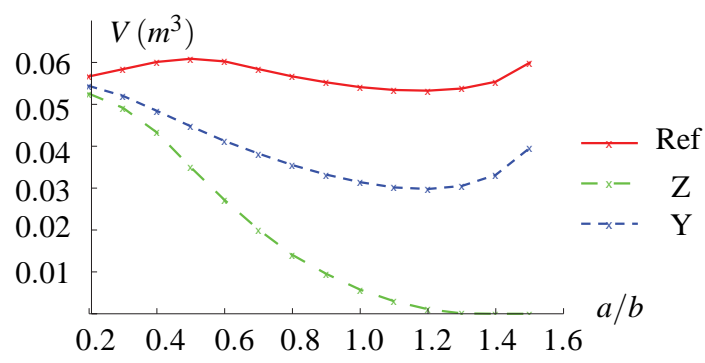

Fig. 14: The workspace volume w.r.t. the ratio of $a / b$, under different orientations

\section{Dexterity Analysis}

The reciprocal of the condition number of the Jacobian matrix, is adopted here [53] to quantify the dexterity; more specifically, we use the condition number based on the Frobenious norm because it is analytical. Since $\mathbf{D}$ is diagonal, its condition number can be readily monitored as the ratio of the largest absolute value to the smallest abosolute value of its nonzero entries, which is well bounded since the limb lengths are designed to be always positive. Moreover, as $\mathbf{J}_{m}$ is orthogonal, it is isotropic. Hence, we only look at matrix $\mathbf{K}$ here, its Frobenius-norm condition number [46], represented as $\kappa_{F}$, being defined as

$$
\begin{aligned}
\kappa_{F}(\mathbf{K}) & =\frac{1}{n} \sqrt{\operatorname{tr}\left(\mathbf{K} \mathbf{K}^{T}\right) \operatorname{tr}\left[\left(\mathbf{K K}^{T}\right)^{-1}\right]} \\
& =\frac{1}{n} \sqrt{\|\mathbf{K}\|_{F}^{2}\left\|\mathbf{K}^{-1}\right\|_{F}^{2}}, \quad n=6
\end{aligned}
$$

Given that the entries of the Jacobian matrices bear different units, we introduce a pertinent characteristic length $L$ [54] to resolve this dimensional inhomogeneity. To this end, we redefine the twist and $\mathbf{K}$ in their dimensionally homogeneous form:

$$
\mathbf{t}_{h}=\left[\begin{array}{c}
\dot{\boldsymbol{\omega}} \\
\dot{\mathbf{c}} / L
\end{array}\right], \quad \mathbf{K}_{h}=\left[\begin{array}{ll}
\left(\mathbf{g}_{1} \times \mathbf{p}_{1}\right)^{T} & L \mathbf{g}_{1}^{T} \\
\left(\mathbf{e}_{1} \times \mathbf{p}_{1}\right)^{T} & L \mathbf{e}_{1}^{T} \\
\left(\mathbf{g}_{2} \times \mathbf{p}_{2}\right)^{T} & L \mathbf{g}_{2}^{T} \\
\left(\mathbf{e}_{2} \times \mathbf{p}_{2}\right)^{T} & L \mathbf{e}_{2}^{T} \\
\left(\mathbf{g}_{3} \times \mathbf{p}_{3}\right)^{T} & L \mathbf{g}_{3}^{T} \\
\left(\mathbf{e}_{3} \times \mathbf{p}_{3}\right)^{T} & L \mathbf{e}_{3}^{T}
\end{array}\right]
$$

The characteristic length is defined as the value $L$ that minimizes the condition number of $\mathbf{K}_{h}$; as reported in a forthcoming paper, the characteristic length of the SDelta robot can be obtained as the radius of the circle circumscribing the equilateraltriangular MP, i.e., as $L=a / \sqrt{3}$. Then, the dexterity is analyzed via a numerical algorithm based on discretization. Four different $Z$-values are chosen, while the dimensions in the other directions are discretized with an interval of $b / 100$; the dexterity distribution is plotted in Figs. 15 and 16 for different orientations ${ }^{12}$. The value $\log _{2}\left(1 / \kappa_{F}\left(\mathbf{K}_{h}\right)\right)$, denoted $\lambda$ for brevity, is plotted vs. $X-Y$, those surfaces with higher altitudes representing a layer with smaller $Z$, i.e., the condition

\footnotetext{
${ }^{11}$ In Designs I and II, we assume that $l_{\max } \approx 2 l_{\min }$ and $l_{\text {ref }} \approx 1 / 2\left(l_{\max }+l_{\min }\right)$, where $l_{\text {ref }}$ represents the limb length at the reference pose; moreover, the reference height of the MP was kept similar in Designs I and II to make a reasonable comparison, which ends up with different $l_{\min }$ and $l_{\max }$.

${ }^{12}$ The dexterity for the reference orientation is similar to that in Fig. 15, thus omitted in the interest of brievity.
} 


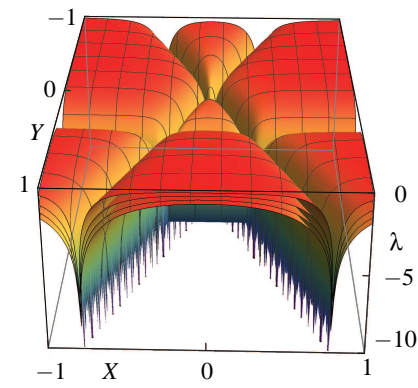

(a)

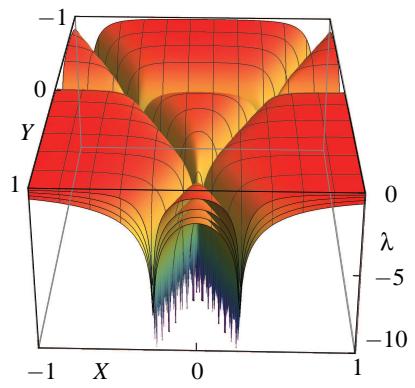

(b)

Fig. 15: The dexterity of the SDelta on the layers of $z=0.3,0.6,0.9,1.2$, with the orientation $\mathbf{e}=[0,0,1]^{T}$ and $\phi=15^{\circ}$ (a) Design I (b) Design II

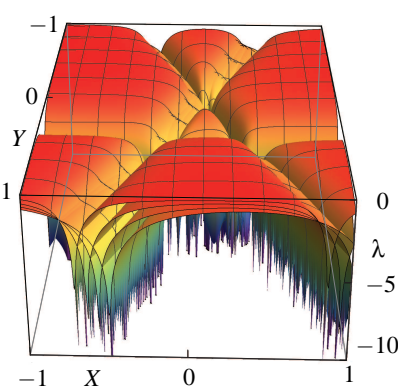

(a)

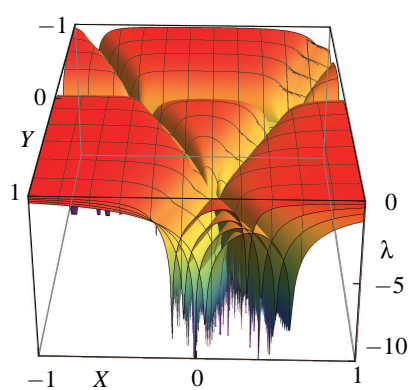

(b)

Fig. 16: The dexterity of the SDelta on the layers of $z=0.3,0.6,0.9,1.2$, with the orientation $\mathbf{e}=[0,1,0]^{T}$ and $\phi=15^{\circ}$ (a) Design I (b) Design II

number generally grows as $Z$ increases. Interestingly, when the normal of the MP triangle is parallel to the $Z$ axis, and the operation point lies in the plane $Z=0$, but not not on the three lines-the intersection of this plane with the three planes of the singularity loci, displayed in Figs. 7 and 8 for two distinct instances, the condition number does not change. The corresponding dexterity surface becomes a plane close to the plane $\lambda=0$, with the condition number $\kappa_{F}$ being roughly equal to 1.06. This means that when the MP moves near the plane of the BP, the condition number is low ${ }^{13}$.

It is found that the dexterity is generally small as long as the posture is not close to a singularity: For the worst case in each orientation (i.e., on the surfaces at the bottom of Figs. 15 and 16, corresponding to $Z=1.2$ ), at least $75 \%$ of the area has a condition number smaller than 8.0 , at least $93 \%$ has a condition number smaller than 32.0 , and at least $99 \%$ has a codition number smaller than 256.0 .

\section{Discussion}

From the above figures for which two sets of arbitrarily chosen design parameters are used, it is apparent that the singularity locus, workspace and dexterity are greatly affected by the ratio $a / b$ and the range of the limb length.

It was found that, when the MP is under the reference orientaion, the singularity locus is the union of three vertical planes, each corresponding to a set of postures under which one of the three points $S_{j}$ lies in the vertical plane passing through the axis of the $j$ th C-Drive, for $j=1,2,3$. As a result, $|a-b / 2|$ must be as large as possible, so that the distance from $S_{j}$ to the vertical plane passing through the axis of the $j$ th C-Drive is as large as possible, the singularity surface becoming farther from the desired workspace region. Furthermore, when the MP rotates about the $Z$ axis, the singularity surface is still the union of three vertical planes. As the MP rotates about an axis other than the $Z$-axis, the singularity surfaces generally show the tendency to both rotate about this axis, and deform in such a way that their shapes become more complex.

Besides the ratio $a / b$, the workspace volume is greatly affected by the range of the limb length: the workspace volume increases as the stroke of each limb increases; moreover, as the average of $l_{\min }$ and $l_{\max }$ increases, the "center" of the workspace region lies higher and the workspace volume generally becomes larger. On the other hand, the dexterity worsens as the "center" of the workspace region lies higher; however, the condition number is generally small enough as long as the operation point is not too close to the singularity surface. As these indices do not obey a monotonic relation, a compromise

\footnotetext{
${ }^{13}$ This plane is not plotted in order to not to hide the details of the other planes
} 
is needed during the optimization process, depending on the application.

The singularity locus sometimes crosses the workspace, which is undesirable; we believe that this is due to the three C-Drive axes becoming coplanar, which yields the three singularity planes at the reference orientation. As a result, other configurations of the three C-Drives may be explored, such as those with three vertical axes, three intersecting orthogonal axes or three skew orthogonal axes. These alternatives have the potential to provide a larger singularity-free workspace and a good dexterity. For example, it can be shown that the layout with three vertical axes can have a larger workspace while keeping a good dexterity, although this platform is mostly suitable for smaller workspaces, due to the possible interference among the three guideways and a large workpiece.

\section{Conclusions}

The subject of this paper is a novel class of parallel robots whose main structure bears a 3-CPS topology, each of its limbs involving, in turn, a parallel substructure, the C-Drive, thereby making it possible to mount all the motors on the base for a three-limb PKM, which leads to a simpler structure, a workspace larger than its six-dof counterparts, and lower inertial load on the system. The actuation scheme realized by the C-drive is symmetric; moreover, the C-drive Jacobian matrix is constant and isotropic, which decouples the analysis of the motor displacements from the three cylindrical motions. The forward displacement analysis also simplifies, yielding a system of three quadratic bivariate polynomials.

We derived the two Jacobian matrices $\mathbf{J}$ and $\mathbf{K}$ for the SDelta Robot, the former as the product of a diagonal matrix $\mathbf{D}$ by a constant isotropic matrix $\mathbf{J}_{m}$. The latter, composed of the Plücker arrays of six lines, was found geometrically.

Type-I singularity analysis is generally straightforward; as for its type-II counterpart, we have derived a relatively simple expression for the singularity condition of the SDelta robot. It can be seen from the derivation that this analysis is applicable to all parallel robots whose six wrench axes per limb intersect pairwise. This simple approach yields one single simple equation involving only the normals of three relevant planes besides constant position vectors, which obviates the calculation of any determinant or passive velocities. This analysis is applicable to numerous parallel robots whose six actuation wrenches bear axes intersecting pairwise, such as three-limb robots that carry a passive spherical joint in each limb, and several simplified Stewart-Gough architectures.

The workspace and dexterity analyses show that the robot has the potential of achieving a larger workspace without sacrificing dexterity. Lastly, several alternative layouts of the C-Drive were proposed, which have the potential to provide a larger singularity-free workspace and good dexterity performance. Further work involves the development of a design optimization algorithm and the exploration of alternative layouts.

\section{Acknowledgements}

The first author would like to acknowledge the support from the McGill Engineering Doctoral Award (MEDA) (Fund Number 90025) and the Chinese Scholarship Council (CSC) (No. 201306020035) for their financial support. The second author acknowledges the support received from NSERC (Canada's Natural Sciences and Engineering Research Council) through Grant No. RGPIN-2015-03864, and from McGill University's James McGill Professorship (Fund Number 100711).

\section{References}

[1] Kong, X., and Gosselin, C., 2007. Type Synthesis of Parallel Mechanisms, Vol. 33. Springer.

[2] Merlet, J.-P., 2006. Parallel Robots, Vol. 128. Springer.

[3] Briot, S., and Khalil, W., 2015. Dynamics of Parallel Robots. Springer.

[4] Stewart, D., 1965. "A platform with six degrees of freedom". Proceedings of the Institution of Mechanical Engineers, 180(1), pp. 371-386.

[5] Fichter, F., 1986. "A Stewart platform-based manipulator - general-theory and practical construction". International Journal of Robotics Research, 5(2), pp. 157-182.

[6] Podhorodeski, R. P., and Pittens, K. H., 1994. "A class of parallel manipulators based on kinematically simple branches". ASME Journal of Mechanical Design, 116(3), pp. 908-914.

[7] Yang, G., Chen, I. M., Chen, W., and Lin, W., 2004. "Kinematic design of a six-dof parallel-kinematics machine with decoupled-motion architecture". IEEE Transactions on Robotics, 20(5), pp. 876-884.

[8] Behi, F., 1988. "Kinematic analysis for a six-degree-of-freedom 3-PRPS parallel mechanism". IEEE Journal of Robotics and Automation, 4(5), pp. 561-565.

[9] Kim, J., Park, F. C., Ryu, S. J., Kim, J., Hwang, J. C., Park, C., and Iurascu, C. C., 2001. "Design and analysis of a redundantly actuated parallel mechanism for rapid machining". IEEE Transactions on Robotics and Automation, 17(4), pp. 423-434.

[10] Sorli, M., Ferraresi, C., Kolarski, M., Borovac, B., and Vukobratovic, M., 1997. "Mechanics of Turin parallel robot". Mechanism and Machine Theory, 32(1), pp. 51-77. 
[11] Chen, C., Gayral, T., Caro, S., Chablat, D., Moroz, G., and Abeywardena, S., 2012. "A six degree of freedom epicyclicparallel manipulator". ASME Journal of Mechanisms and Robotics, 4(4), p. 041011.

[12] Liu, X. J., and Wang, J. S., 2003. "Some new parallel mechanisms containing the planar four-bar parallelogram". International Journal of Robotics Research, 22(9), pp. 717-732.

[13] Monsarrat, B., and Gosselin, C. M., 2003. "Workspace analysis and optimal design of a 3-leg 6-dof parallel platform mechanism”. IEEE Transactions on Robotics and Automation, 19(6), pp. 954-966.

[14] Jin, Y., Chen, I. M., and Yang, G. L., 2009. "Kinematic design of a family of 6-dof partially decoupled parallel manipulators". Mechanism and Machine Theory, 44(5), pp. 912-922.

[15] Azulay, H., Mahmoodi, M., Zhao, R., Mills, J. K., and Benhabib, B., 2014. "Comparative analysis of a new 3xPPRS parallel kinematic mechanism". Robotics and Computer-Integrated Manufacturing, 30(4), pp. 369-378.

[16] Fu, J., Gao, F., Pan, Y., and Du, H., 2015. "Forward kinematics solutions of a special six-degree-of-freedom parallel manipulator with three limbs". Advances in Mechanical Engineering, 7(5), p. 1687814015582118.

[17] Wu, Y. N., and Gosselin, C. M., 2004. "Synthesis of reactionless spatial 3-dof and 6-dof mechanisms without separate counter-rotations". International Journal of Robotics Research, 23(6), pp. 625-642.

[18] Wampler, C., Morgan, A., and Sommese, A., 1990. "Numerical continuation methods for solving polynomial systems arising in kinematics". Journal of Mechanical Design, 112(1), pp. 59-68.

[19] Faugère, J.-C., and Lazard, D., 1995. "Combinatorial classes of parallel manipulators". Mechanism and Machine Theory, 30(6), pp. 765-776.

[20] Innocenti, C., and Parenticastelli, V., 1990. "Direct position analysis of the stewart platform mechanism". Mechanism and Machine Theory, 25(6), pp. 611-621.

[21] Gan, D., Dias, J., and Seneviratne, L., 2016. "Unified kinematics and optimal design of a 3rrps metamorphic parallel mechanism with a reconfigurable revolute joint". Mechanism and Machine Theory, 96, pp. 239-254.

[22] Merlet, J. P., 2004. "Solving the forward kinematics of a gough-type parallel manipulator with interval analysis". International Journal of Robotics Research, 23(3), pp. 221-235.

[23] Gibson, C. G., and Hunt, K. H., 1990. "Geometry of screw systems-1. screws - genesis and geometry". Mechanism and Machine Theory, 25(1), pp. 1-10.

[24] Merlet, J. P., 1989. "Singular configurations of parallel manipulators and Grassmann geometry". International Journal of Robotics Research, 8(5), pp. 45-56.

[25] Park, F., and Kim, J. W., 1999. "Singularity analysis of closed kinematic chains". Journal of Mechanical Design, 121(1), pp. 32-38.

[26] Zlatanov, D., Fenton, R. G., and Benhabib, B., 1998. "Identification and classification of the singular configurations of mechanisms". Mechanism and Machine Theory, 33(6), pp. 743-760.

[27] Bohigas, O., Zlatanov, D., Ros, L., Manubens, M., Porta, J. M., and IEEE, 2012. "Numerical computation of manipulator singularities". 2012 IEEE International Conference on Robotics and Automation (ICRA), pp. 1351-1358.

[28] Wen, J. T., and O'Brien, J. F., 2003. "Singularities in three-legged platform-type parallel mechanisms". IEEE Transactions on Robotics and Automation, 19(4), pp. 720-726.

[29] Ben-Horin, P., and Shoham, M., 2006. "Singularity condition of six-degree-of-freedom three-legged parallel robots based on Grassmann-cayley algebra". IEEE Transactions on Robotics, 22(4), pp. 577-590.

[30] Ben-Horin, P., and Shoham, M., 2006. "Singularity analysis of a class of parallel robots based on Grassmann-cayley algebra". Mechanism and Machine Theory, 41(8), pp. 958-970.

[31] Kong, X. W., and Gosselin, C. M., 2001. "Uncertainty singularity analysis of parallel manipulators based on the instability analysis of structures". International Journal of Robotics Research, 20(11), pp. 847-856.

[32] Gosselin, C., and Angeles, J., 1990. "Singularity analysis of closed-loop kinematic chains". IEEE Transactions on Robotics and Automation, 6(3), pp. 281-290.

[33] Bonev, I. A., and Gosselin, C. M., 2000. "A geometric algorithm for the computation of the constant-orientation workspace of 6-rus parallel manipulators". In Proceedings of the 2000 ASME Design Engineering Technical Conferences.

[34] Masory, O., and Wang, J., 1995. "Workspace evaluation of stewart platforms". Advanced Robotics, 9(4), pp. 443-461.

[35] Majid, M. Z. A., Huang, Z., and Yao, Y. L., 2000. "Workspace analysis of a six-degrees of freedom, three-prismaticprismatic-spheric-revolute parallel manipulator". International Journal of Advanced Manufacturing Technology, 16(6), pp. 441-449.

[36] Dash, A. K., Chen, I. M., Yeo, S. H., and Yang, G. L., 2005. "Workspace generation and planning singularity-free path for parallel manipulators". Mechanism and Machine Theory, 40(7), pp. 776-805.

[37] Jo, D. Y., and Haug, E. J., 1989. "Workspace analysis of closed loop mechanisms with unilaterial constraints". In Advances in Design Automation - 1989, September 17, 1989 - September 21, 1989, Vol. 19-3 of American Society of Mechanical Engineers, Design Engineering Division (Publication) DE, Publ by American Soc of Mechanical Engineers (ASME), pp. 53-60.

[38] Adkins, F. A., and Haug, E. J., 1997. “Operational envelope of a spatial stewart platform”. ASME Journal of Mechanical 
Design, 119(2), pp. 330-332.

[39] Haug, E., Luh, C.-M., Adkins, F., and Wang, J.-Y., 1996. "Numerical algorithms for mapping boundaries of manipulator workspaces". ASME Journal of Mechanical Design, 118(2), pp. 228-234.

[40] Merlet, J. P., 1999. "Determination of 6d workspaces of gough-type parallel manipulator and comparison between different geometries". International Journal of Robotics Research, 18(9), pp. 902-916.

[41] Patel, S., and Sobh, T., 2014. "Manipulator performance measures-A comprehensive literature survey". Journal of Intelligent \& Robotic Systems, pp. 1-24.

[42] Moreno, H. A., Saltaren, R., Carrera, I., Puglisi, L., and Aracil, R., 2012. "Performance indices for robotic manipulators: A review of the state of the art". Revista Iberoamericana de Automatica e Informatica Industrial, 9(2), pp. 111-122.

[43] Harada, T., Friedlaender, T., and Angeles, J., 2014. "The development of an innovative two-dof cylindrical drive: Design, analysis and preliminary tests". In 2014 IEEE International Conference on Robotics and Automation (ICRA), pp. 6338-6344.

[44] Nanua, P., Waldron, K. J., and Murthy, V., 1990. “Direct kinematic solution of a stewart platform”. IEEE Transactions on Robotics and Automation, 6(4), pp. 438-444.

[45] Hunt, K. H., 1986. "Special configurations of robot-arms via screw theory". Robotica, 4, pp. $171-179$.

[46] Angeles, J., 2014. Fundamentals of Robotic Mechanical Systems. Theory, Methods, Algorithms, 4 ed. Springer, New York.

[47] Burdick, J. W., 1995. "A classification of 3R regional manipulator singularities and geometries". Mechanism and Machine Theory, 30(1), pp. 71-89.

[48] Notash, L., 1998. "Uncertainty configurations of parallel manipulators". Mechanism and Machine Theory, 33(1-2), pp. 123-138.

[49] Yang, G., Chen, I.-M., Lin, W., and Angeles, J., 2001. "Singularity analysis of three-legged parallel robots based on passive-joint velocities". IEEE Transactions on Robotics and Automation, 17(4), pp. 413-422.

[50] Downing, D. M., Samuel, A. E., and Hunt, K. H., 2002. "Identification of the special configurations of the octahedral manipulator using the pure condition”. International Journal of Robotics Research, 21(2), pp. 147-159.

[51] Ebert-Uphoff, I., Lee, J. K., and Lipkin, H., 2002. "Characteristic tetrahedron of wrench singularities for parallel manipulators with three legs". Proceedings of the Institution of Mechanical Engineers Part C-Journal of Mechanical Engineering Science, 216(1), pp. 81-93.

[52] FarzanehKaloorazi, M., Masouleh, M. T., and Caro, S., 2016. "Collision-free workspace of parallel mechanisms based on an interval analysis approach". Robotica, pp. 1-14.

[53] Angeles, J., and López-Cajún, C. S., 1992. "Kinematic isotropy and the conditioning index of serial robotic manipulators". International Journal of Robotics Research, 11(6), pp. 560-571.

[54] Zanganeh, K. E., and Angeles, J., 1997. "Kinematic isotropy and the optimum design of parallel manipulators". The International Journal of Robotics Research, 16(2), pp. 185-197. 


\section{List of Figure Captions}

Fig. 1: Topology of the SDelta robot

Fig. 2: Topology of the C-drive

Fig. 3: Architecture of the SDelta Robot

Fig. 4: An embodiment of the C-drive

Fig. 5: Notation for the kinematic chain of the $j$ th limb of the SDelta Robot

Fig. 6: The reference posture of the MP

Fig. 7: The workspace and singularity of the SDelta under the reference orientation (a) Design I (b) Design II

Fig. 8: The workspace and singularity of the SDelta with the orientation $\mathbf{e}=[0,0,1]^{T}$ and $\phi=15^{\circ}$ (a) Design I (b)

Design II

Fig. 9: The workspace and singularity of the SDelta with the orientation $\mathbf{e}=[0,1,0]^{T}$ and $\phi=15^{\circ}$ (a) Design I (b)

\section{Design II}

Fig. 10: Illustration of the workspace formulation based on the geometric method

Fig. 11: The workspace of the Sdelta under the reference orientation (a) Design I (b) Design II

Fig. 12: The workspace of the SDelta with the orientation $\mathbf{e}=[0,0,1]^{T}$ and $\phi=15^{\circ}$ (a) Design I (b) Design II

Fig. 13: The workspace of the SDelta with the orientation $\mathbf{e}=[0,1,0]^{T}$ and $\phi=15^{\circ}$ (a) Design I (b) Design II

Fig. 14: The workspace volume w.r.t. the ratio of $a / b$, under different orientations

Fig. 15: The dexterity of the SDelta on the layers of $z=0.3,0.6,0.9,1.2$, with the orientation $\mathbf{e}=[0,0,1]^{T}$ and $\phi=15^{\circ}$

(a) Design I (b) Design II

Fig. 16: The dexterity of the SDelta on the layers of $z=0.3,0.6,0.9,1.2$, with the orientation $\mathbf{e}=[0,1,0]^{T}$ and $\phi=15^{\circ}$ (a) Design I (b) Design II 